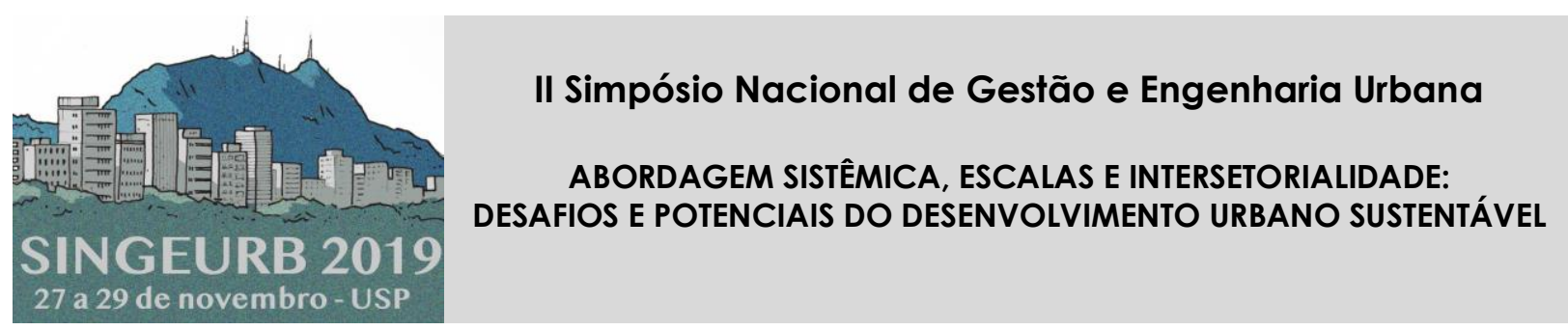

\title{
O discurso de sustentabilidade nos Planos Diretores de cidades médias paulistas ${ }^{1}$
}

\section{The sustainability speech in the Municipal Director Plans in medium sized cities of São Paulo state}

\author{
Amanda Carvalho Maia'; Gisela Cunha Viana Leonelli²
}

1 Programa de Pós-Graduação em Arquitetura, Tecnologia e Cidade. Universidade Estadual de Campinas. Rua Saturnino de Brito, 224 - Cidade Universitária, Campinas - SP, Brasil. amandacmaial@gmail.com 2 Programa de Pós-Graduação em Arquitetura, Tecnologia e Cidade. Universidade Estadual de Campinas. gisela@fec.unicamp.br.

\begin{abstract}
RESUMO
O mote de desenvolvimento sustentável emerge como um jargão nos discursos institucionais de ordenamento territorial. Esta atribuição se insere em um contexto de comprometimento dos órgãos públicos com a responsabilidade ambiental afirmada em pactos internacionais como Agenda 21 e Conferências Habitat I, II e III. No âmbito nacional, a aprovação do Estatuto da Cidade, principal documento jurídico de controle da expansão urbana, tem como embasamento para suas diretrizes a sustentabilidade econômica, política e social. Apesar da importância de apreensão dos múltiplos discursos acerca da sustentabilidade, o enfoque do trabalho situa-se na identificação e análise de sua utilização pelos Planos Diretores de cidades médias do Estado de São Paulo. Como metodologia, adotou-se a identificação do termo "sustentabilidade" no Estatuto da Cidade e nos Planos Diretores municipais em nove cidades paulistas de porte médio: Presidente Prudente, São José do Rio Preto, Bauru, Franca, Limeira, Piracicaba, Ribeirão Preto, São Carlos e Araraquara. Como resultado, constatou-se a assimilação de palavras relacionadas a sustentabilidade, utilizando o tema como principal eixo de fundamentação das diretrizes do Plano Diretor. Entretanto, não estabelecem estratégias efetivas e autoaplicáveis.
\end{abstract}

Palavras-chave: discurso de sustentabilidade, planos diretores municipais, cidades médias paulistas.

\section{ABSTRACT}

The subject of sustainable development emerges as a jargon in the institutional discourses of land use planning. This attribution is part of a commitment by public agencies to the environmental concerns affirmed in international pacts such as Agenda 21, Habitat I, II and III Conferences. At the national level, the approval of the City Statute, the main legal document on the control of urban expansion, is based on its guidelines for economic, political and social

\footnotetext{
${ }^{1}$ MAIA, Amanda Carvalho; LEONELLI, Gisela Cunha Viana. O discurso de sustentabilidade nos Planos Diretores de cidades médias paulistas. In: II SIMPÓSIO NACIONAL DE GESTÃO E ENGENHARIA URBANA: SINGEURB, 2019, São Paulo. Anais... Porto Alegre: ANTAC, 2019.
} 
sustainability. Despite the importance of apprehending multiple discourses about sustainability, the focus of the work is on the identification and analysis of its use by the Master Plans of medium-sized cities in the State of São Paulo. As a methodology, The term of sustainability was verified in the City Statute, as well as the demand in the Municipal Director Plans in nine cities: Presidente Prudente, São José do Rio Preto, Bauru, Franca, Limeira, Piracicaba, Ribeirão Preto, São Carlos e Araraquara. As a result, it was verified the assimilation of words related to sustainability, using the theme as the main axis of foundation of the guidelines of the Master Plan. However, they do not establish effective and self-enforcing strategies.

Keywords: sustainability speech, municipal director plans, medium sized cities of São Paulo state.

\section{INTRODUÇÃO}

O termo sustentabilidade tornou-se oportuno em debates de todas as naturezas, estando presente em contextos diversos com múltiplos sentidos. De acordo com Acselrad (1999) a noção de sustentabilidade se relaciona às práticas, ou seja, apoiado na sua assimilação como algo bom, consensual e desejável coloca-se como elo de divisão para elencar as atividades consideradas inapropriadas.

A disseminação do discurso de sustentabilidade iniciou-se na década de 1970, mediante reconhecimento de movimentos sociais ecológicos, encontro entre técnicos e cientistas como o Clube de Roma e nas conferências internacionais promovidas pela ONU para discutir questões ambientais aliadas ao desenvolvimento econômico. A primeira aparição aconteceu na Conferência de Estocolmo, em 1972, com enfoque técnico e alarmista sobre crescimento populacional urbano e suas implicações na degradação ambiental (GUIMARÃES, 1997). A conceitualização mais expressiva do "desenvolvimento sustentável" despontou do relatório da Comissão Brundtland (1983) que propôs vincular o crescimento econômico a preservação ambiental a partir da ênfase econômica e tecnológica. Outra importante caracterização do "desenvolvimento sustentável" foi proposta por Ignacy Sachs (1986) através da percepção do que o autor denominou de "Ecodesenvolvimento" que recomenda, além da articulação entre a preservação ambiental e a economia, a equidade social. Para Sachs, o combate às desigualdades sociais e a autonomia da população dos então chamados "países de terceiro mundo" é primordial (LIMA, 1997).

No contexto das cidades, a noção de sustentabilidade predominante se assimila ao meio técnico no tocante à racionalidade ecológica e, especialmente, energética (ACSELRAD, 1997). Assim, a cidade sustentável seria aquela que, cogitando o mesmo padrão de consumo, minimiza o uso de recursos naturais, energia, matéria-prima, produção de rejeitos e destinação de resíduos sólidos, almejando uma alta performance com o ambiente construído.

Apesar da importância de apreensão dos múltiplos discursos acerca da sustentabilidade, o enfoque do trabalho situa-se na análise da sua utilização pelos Planos Diretores de cidades médias do Estado de São Paulo2. Quanto ao Plano Diretor, constitui-se como o protagonista na orientação do crescimento territorial das cidades brasileiras. Este, ao operar em escala local, deve seguir as diretrizes e objetivos estabelecidos pelas normativas nacionais, no caso, o Estatuto da Cidade (Lei ${ }^{\circ}$ 10.257) e a Constituição Federal que preveem como atribuição máxima do urbano, o cumprimento da função social da propriedade.

Metodologicamente, foi realizada uma investigação na letra da lei do Estatuto da Cidade que prevê a orientação de cidades sustentáveis e, ao tomar como base para a elaboração dos Planos Diretores, a análise da legislação municipal. Em diante, sucederam averiguações dos Planos diretores de nove municípios paulistas para identificar de que forma o discurso de sustentabilidade é incorporado aos mesmos, a saber: Presidente Prudente, São José do Rio Preto, Bauru, Franca, Limeira, Piracicaba, Ribeirão Preto, São Carlos e Araraquara. O recorte

2 Estes municípios atuam como polos regionais de atividades e serviços e exercem papel de intermediação entre as localidades pequenas e as maiores (SPOSITO, 2006). 
territorial é embasado no levantamento do REGIC (IBGE, 2007) que elenca a hierarquia da rede de cidades em todo Brasil. Foram selecionadas cidades paulistas na categoria de Capital Regional e Centro Sub-regional.

O ensaio levanta questões, não sendo resultado de pesquisas concluídas e, sim, de inquietações iniciais que poderá orientar outras explorações e, possivelmente, novas lentes para a análise da temática de sustentabilidade na legislação urbanística. Ademais, pode auxiliar na escolha do objeto empírico para estudos de caso, uma vez que é decorrência de levantamentos de diversos municípios.

\title{
2. O ESTATUTO DA CIDADE E O PLANEJAMENTO URBANO SUSTENTÁVEL
}

No âmbito da política urbana, a noção de sustentabilidade se estende à percepção de eficiência energética e preservação ambiental, posicionando-a como justiça social. Para tanto, o Estatuto da Cidade, define em seus objetivos:

\begin{abstract}
garantia do direito a cidades sustentáveis, entendido como o direito à terra urbana, à moradia, ao saneamento ambiental, à infraestrutura urbana, ao transporte e aos serviços públicos, ao trabalho e ao lazer, para as presentes e futuras gerações. (Lei $n^{\circ} 10.257$, art. $n^{\circ} 2, \S 1^{\circ}$ )

adoção de padrões de produção e consumo de bens e serviços e de expansão urbana compatíveis com os limites da sustentabilidade ambiental, social e econômica do Município e do território sob sua área de influência. (Lei $\mathrm{n}^{\circ}$ 10.257, art. $\mathrm{n}^{\circ} 2, \S 8^{\circ}$ )
\end{abstract}

Estas são as únicas passagens do Estatuto da Cidade que se referem explicitamente ao termo "sustentabilidade". Ademais, são muitos os instrumentos de gestão urbana que procuram assegurar a função social da propriedade urbana e a garantia ao direito de cidades sustentáveis.

\section{O EMPREGO DO TERMO SUSTENTABILIDADE NOS PLANOS DIRETORES}

O quadro 1 sintetiza o uso do termo de sustentabilidade nos Planos Diretores Municipais. Todos os municípios incorporaram o discurso de sustentabilidade sendo que a maior parte (Presidente Prudente, Piracicaba, Ribeirão Preto e São Carlos) não define o que é sustentabilidade, apesar de adotar o termo como objetivo e diretriz. As cidades de São José do Rio Preto, Franca e Limeira utilizam da definição presente no Estatuto da Cidade que considera o acesso à moradia, serviços e infraestrutura como sinônimo de cidade sustentável. Já Bauru emprega o conceito apenas quando trata do rural.

No geral, o termo de sustentabilidade aparece nos objetivos e diretrizes dos PD's, seja no início do texto, seja nos capítulos sobre moradia, e mobilidade não sendo agregado nos instrumentos de política urbana. A nomenclatura também é comum nos títulos e subtítulos de Fundos financeiros e Conselhos. O consenso é o seu emprego para se referir à preservação ambiental ou associado ao ecossistema, principalmente sobre a racionalidade do uso dos recursos naturais. Todavia, a menção ocorre apenas em referência às generalidades.

O PD de São José do Rio Preto, por exemplo, dispõe da palavra em 41 ocasiões, pois está empregada no título do PD. Fora isso, o uso da sustentabilidade é inócuo e genérico. No caso de Presidente Prudente, a citação da sustentabilidade é contraditória, uma vez que implica que o desenvolvimento sustentável pode atrapalhar o desenvolvimento socioeconômico.

O município de Araraquara é o caso mais emblemático, pois incorpora o tema da sustentabilidade em todo o texto como nos objetivos, diretrizes e nos instrumentos de política urbana, como o parcelamento, parcelamento edificação e uso compulsório, à geração de renda (rural e urbano), dentre outros. 
Quadro 1 - Emprego do termo sustentabilidade nos planos diretores de cidades médias paulistas

\begin{tabular}{|c|c|}
\hline \multicolumn{2}{|r|}{ Presidente Prudente (Lei n²30/2018) } \\
\hline $\begin{array}{l}\text { Instâncias da palavra } \\
\text { sustentável }\end{array}$ & 9 \\
\hline Conceito de sustentabilidade & Não consta \\
\hline Presença do termo & $\begin{array}{l}\text { Objetivos, princípios, padrões de ocupação (limite ambiental), } \\
\text { mobilidade urbana, Parque, objetos construídos. }\end{array}$ \\
\hline Funções discursivas & $\begin{array}{l}\text { O termo se refere a princípios, objetivos e generalidades. O plano e } \\
\text { demais leis urbanísticas não mencionam o termo em instrumentos e } \\
\text { medidas autoaplicáveis }\end{array}$ \\
\hline Contradições & $\begin{array}{l}\text { A menção no artigo } 7^{\circ} \text { pressupõe incompatibilidade entre } \\
\text { desenvolvimento sustentável e desenvolvimento socioeconômico. } \\
\text { "garantia de que o desenvolvimento sustentável da economia e da } \\
\text { estrutura física não venham inviabilizar o desenvolvimento } \\
\text { socioeconômico do município" (Art. } 7, \S 5^{\circ} \text { ). }\end{array}$ \\
\hline \multicolumn{2}{|r|}{ São José do Rio Preto (Lei n $224 / 2006$ ) } \\
\hline $\begin{array}{l}\text { Instâncias da palavra } \\
\text { sustentável }\end{array}$ & 43, sendo que 31 se referem ao título do Plano Diretor \\
\hline Conceito de sustentabilidade & $\begin{array}{l}\text { "Sustentabilidade urbana é o desenvolvimento local socialmente } \\
\text { justo, ambientalmente equilibrado e economicamente viável, } \\
\text { visando garantir qualidade de vida para a presente e as futuras } \\
\text { gerações" (Art. 6) }\end{array}$ \\
\hline Presença do termo & Título, subtítulos, princípios e objetivos \\
\hline Funções discursivas & $\begin{array}{l}\text { Generalidades. Só é específico trata do fundo de desenvolvimento } \\
\text { sustentável }\end{array}$ \\
\hline \multicolumn{2}{|r|}{ Bauru (Lei $\left.n^{\circ} 5631 / 2008\right)$} \\
\hline $\begin{array}{l}\text { Instâncias da palavra } \\
\text { sustentável }\end{array}$ & - $1-1,-1$ \\
\hline Conceito de sustentabilidade & $\begin{array}{l}\text { O plano só define o que é desenvolvimento sustentável para a área } \\
\text { rural: "àquele economicamente viável, socialmente justo, } \\
\text { ambientalmente correto e que respeita os aspectos culturais e } \\
\text { históricos" (Art. 213). }\end{array}$ \\
\hline Presença do termo & $\begin{array}{l}\text { Subtítulos, objetivos, princípios, diretriz de mobilidade, uso e } \\
\text { ocupação do solo, segundo limites de suporte. }\end{array}$ \\
\hline Funções discursivas & $\begin{array}{l}\text { Generalidades. O termo é utilizado em menção as áreas rurais ou de } \\
\text { proteção ambiental. }\end{array}$ \\
\hline \multicolumn{2}{|r|}{ Franca (Lei $n^{\circ}$ 50/2003) } \\
\hline $\begin{array}{l}\text { Instâncias da palavra } \\
\text { sustentável }\end{array}$ & 要 \\
\hline Conceito de sustentabilidade & $\begin{array}{l}\text { "garantia do direito a cidade sustentável, entendido como direito à } \\
\text { terra urbana, à moradia, ao saneamento ambiental, à infraestrutura } \\
\text { urbana, ao transporte e aos serviços públicos, ao trabalho e ao lazer, } \\
\text { para as presentes e futuras gerações" (Art.3) }\end{array}$ \\
\hline Presença do termo & Objetivos \\
\hline \multicolumn{2}{|r|}{ Limeira (Lei n 442/2009) } \\
\hline $\begin{array}{l}\text { Instâncias da palavra } \\
\text { sustentável }\end{array}$ & ( \\
\hline Conceito de sustentabilidade & $\begin{array}{l}\text { "garantia do direito a uma cidade sustentável, entendido como } \\
\text { direito à terra urbana, à moradia, ao saneamento ambiental, à } \\
\text { infraestrutura urbana, ao transporte e aos serviços públicos, ao } \\
\text { trabalho e ao lazer, para as presentes e futuras gerações" (Art. 5) }\end{array}$ \\
\hline Presença do termo & Objetivos e diretrizes, limites de uso do solo \\
\hline Funções discursivas & Racionalização dos recursos naturais e ambiental \\
\hline \multicolumn{2}{|r|}{ Piracicaba (Lei 186/2006) } \\
\hline $\begin{array}{l}\text { Instâncias da palavra } \\
\text { sustentável }\end{array}$ & 5 \\
\hline Conceito de sustentabilidade & Não Consta \\
\hline Presença do termo & Objetivos e diretrizes de mobilidade, preservação e moradia \\
\hline Funções discursivas & Generalidades nas diretrizes \\
\hline & Ribeirão Preto (Lei n² 2866/2018) \\
\hline
\end{tabular}




\begin{tabular}{|c|c|}
\hline $\begin{array}{l}\text { Instâncias da palavra } \\
\text { sustentável }\end{array}$ & 33 \\
\hline Conceito de sustentabilidade & Não Consta \\
\hline Presença do termo & $\begin{array}{l}\text { Objetivos, e diretrizes de mobilidade, desenvolvimento econômico, } \\
\text { moradia, centros tecnológicos, racionalidade do uso dos recursos } \\
\text { naturais, preservação ambiental, limites e capacidade do solo com } \\
\text { suporte na infraestrutura }\end{array}$ \\
\hline Funções discursivas & $\begin{array}{l}\text { Aparece na maioria das diretrizes e, apesar de enfocar no ambiental } \\
\text { e econômico, cita outras esferas. }\end{array}$ \\
\hline \multicolumn{2}{|r|}{ São Carlos (Lei n¹8.053/2018) } \\
\hline $\begin{array}{l}\text { Instâncias da palavra } \\
\text { sustentável }\end{array}$ & 13 \\
\hline Conceito de sustentabilidade & Não Consta \\
\hline Presença do termo & $\begin{array}{l}\text { Objetivos, uso de fontes renováveis, racionalização do uso dos } \\
\text { recursos hídricos, atividades econômicas, atividades rurais proteção } \\
\text { ambiental, uso do imóvel (instrumentos) }\end{array}$ \\
\hline Funções discursivas & $\begin{array}{l}\text { Generalidades nos objetivos e diretrizes, mas abrange o discurso } \\
\text { além do tema ambiental, colocando a sustentabilidade econômica, } \\
\text { de acesso a mobilidade, diversidade de atividades e, utiliza o termo } \\
\text { em um instrumento de política urbana. }\end{array}$ \\
\hline \multicolumn{2}{|r|}{ Araraquara (Lei $n^{\circ} 850 / 2014$ ) } \\
\hline $\begin{array}{l}\text { Instâncias da palavra } \\
\text { sustentável }\end{array}$ & 103 \\
\hline Conceito de sustentabilidade & $\begin{array}{l}4 \text { ocasiões, sendo uma no texto de lei e outras } 3 \text { no glossário: } \\
\text { "Entende-se como desenvolvimento sustentável um processo de } \\
\text { modificação da biosfera e do ambiente construído no qual a } \\
\text { utilização de recursos, a direção de investimentos, a orientação do } \\
\text { desenvolvimento tecnológico e a mudança institucional se } \\
\text { harmonizam a fim de atender a qualidade de vida e as aspirações } \\
\text { humanas das gerações presentes e futuras (Artigo 3, parágrafo } \\
\text { único). }\end{array}$ \\
\hline Presença do termo & $\begin{array}{l}\text { Objetivos, princípios, estratégias, participação democrática, uso e } \\
\text { aproveitamento seguindo padrões suporte do solo, distribuição dos } \\
\text { equipamentos e serviços públicos, expansão econômica, produção } \\
\text { familiar (rural), geração de emprego e renda, densidade adequada, } \\
\text { compacidade e continuidade territorial e política de moradia e } \\
\text { mobilidade. }\end{array}$ \\
\hline Funções discursivas & O termos está presente em todo o texto \\
\hline
\end{tabular}

\section{CONCLUSÕES}

O discurso de sustentabilidade é tendência nos Planos Diretores municipais. Esse importante documento de ordenamento territorial reúne o tema, sobretudo, nos objetivos e diretrizes, sem integrar as propostas, traduzindo como genérico. Ademais, a maioria sequer define o que considera como sustentável. A exceção é o município de Araraquara, que faz menção ao tema em todo o texto.

Este levantamento é inicial e sugere caminhos para a análise da sustentabilidade em planos diretores, tema que deve ser estudado com maior ênfase a fim de verificar a coerência entre o discurso e a prática.

\section{REFERÊNCIAS}

ACSELRAD, Henri. Discursos da sustentabilidade urbana. Revista Brasileira de Estudos Urbanos e Regionais, [s.l.], v. 1, n. 1, p.79-90, 31 maio 1999. Disponível em: <http://rbeur.anpur.org.br/rbeur/article/view/27>. Acesso 10 abr. 2019. 
ARARAQUARA. Lei Complementar $\mathbf{n}^{\circ} \mathbf{8 5 0}$ de 11 de fevereiro de 2014. Estabelece a Revisão do Plano Diretor de Desenvolvimento e Política Ambiental de Araraquara - PDPUA, revoga a Lei Complementar $n^{\circ}$ 350/05 e alterações e institui o Plano Diretor de Desenvolvimento e Política Ambiental de Araraquara - PDDPA, conforme estabelece o parágrafo $3^{\circ}$ do artigo 40 do Estatuto da Cidade. Disponível em: < http://www.camara-

arq.sp.gov.br/Siave/arquivo? ld=123213>. Acesso em: 15 abr. 2019.

BAURU. Lei Municipal $\mathbf{n}^{\circ}$ 5631, de 22 de agosto de 2008. Institui o Plano Diretor Participativo do Município de Bauru. Disponível em: <https://www.bauru.sp.leg.br/legislacao/legislacaomunicipal>. Acesso em: 15 abr. 2019.

BIAZIN, C. C.; GODOY, A. M. G. O selo verde: uma nova exigência internacional para as organizações. In: ENCONTRO NACIONAL DE ENGENHARIA DE PRODUÇÃO, 20., 2000, São Paulo. Anais... São Paulo: ABEPRO, 2000.

FRANCA. Lei Complementar $\mathbf{n}^{\circ}$ 50, de 17 de janeiro de 2003. Institui o Plano Diretor do município de Franca e dá outras providências. Disponível em: <

https://franca.sp.leg.br/legislacao/lei-complementar-no-50-de-17-de-janeiro-de-2003>. Acesso em: 15 abr. 2019.

GUIMARÃES, Roberto. Desenvolvimento sustentável: da retórica à formulação de políticas públicas. In: BECKER, Berta e MIRANDA, Mariana (orgs.). A Geografia Política do Desenvolvimento Sustentável. Rio de Janeiro: Ed. UFRJ, 1997. p. 13-44.

INSTITUTO BRASILEIRO DE GEOGRAFIA E ESTATÍSTICA. Regiōes de Influência das Cidades. 2007. Disponível em: < https://ww2.ibge.gov.br/home/geociencias/geografia/regic.shtm>. Acesso 10 abril de 2019.

LIMA, G. C. O debate da sustentabilidade na sociedade insustentável. Revista Política \&Trabalho, n 13: 201-222, João Pessoa: PPGS/UFPB, setembro/1997.

LIMEIRA. Lei Complementar $\mathbf{n}^{\circ} \mathbf{4 4 2}$, de 12 de janeiro de 2009. Dispõe sobre o plano diretor territorial-ambiental do município de limeira e dá outras providências. Disponível em: <http://consulta.limeira.sp.leg.br/Documentos/Pesquisa?Pesquisa=Simples\&id=80\&pagina=1 $\&$ Modulo $=8 \&$ Documento $=0 \&$ Numeracao=Documento \&Numerolnicial $=442 \&$ Anolnicial $=2009>$ . Acesso em: 23 mar. 2019.

PIRACICABA. Lei Complementar $\mathbf{n}^{\circ} \mathbf{1 8 6}$, de 10 de outubro de 2016. Aprova o Plano Diretor de Desenvolvimento do município de Piracicaba, cria conselho da cidade, revoga a lei complementar n $46 / 95$ e suas alterações e dá outras providências. Disponível em: < http://siave.camarapiracicaba.sp.gov.br/arquivo?ld=225176>. Acesso em: 23 mar. 2019.

PRESIDENTE PRUDENTE. Lei Complementar $\mathbf{n}^{\circ} \mathbf{2 3 0}$ de 20 de dezembro de 2018. Dispõe sobre a Lei do Plano Diretor do Município de Presidente Prudente e revoga a Lei Complementar ${ }^{\circ}$ 151/2008. Disponível em: < https://www.camarapprudente.sp.gov.br/site/Legislacao/ ?pag=TORNPU9UZZI PVFK9TORnPU9EWT1OAMC9TI dRPU9HRTI PVGM9TOdVPU9HTTI PVEU9TOdVP U4YSTIPVFE9T1 dFPVIUUTO=\&set=2>. Acesso em: 15 abr. 2019.

RIBEIRÃO PRETO. Lei Complementar $\mathbf{n}^{\circ}$ 2866, de 27 de abril de 2018. Ribeirão Preto, Disponível em: <https://www.ribeiraopreto.sp.gov.br/J321/pesquisa.xhtmlelei=25704>. Acesso em: 5 mar. 2018.

SACHS, I. Ecodesenvolvimento: crescer sem destruir. São Paulo: Vértice, 1986.

SÃO CARLOS. Lei ordinária n 18056, de 19 de dezembro de 2016. Estabelece o Plano Diretor do Município de São Carlos e dá outras providências. Disponível em: < https://file.gtp.net.br/lei/arquivo/70792/CODIGOLEl_34772.pdf> Acesso em: 23 mar. 2019. 
SÃO JOSÉ DO RIO PRETO. Lei Complementar n²24, aprovada em 06 de outubro de 2006. Dispõe dobre o Plano Diretor de Desenvolvimento Sustentável de São José do Rio Preto.

Disponível em: https://leismunicipais.com.br/a2/sp/s/sao-jose-do-rio-preto/leicomplementar/2006/23/224/lei-complementar-n-224-2006-dispoe-sobre-o-plano-diretor-dedesenvolvimento-sustentavel-de-sao-jose-do-rio-preto? q=224>. Acesso em: 13 abr. 2019. 